\title{
SIMULTANEOUS DETERMINATION OF CONTENTS OF THREE ACTIVE COMPONENTS IN JIEJIA TINCTURE BY HPLC METHOD
}

\author{
Li Kun ${ }^{1} \dagger$, Wang Fei ${ }^{2} \uparrow$, Diao Yun-Peng ${ }^{3}$, Pan Xiao-qiu ${ }^{3}$, E Yun-Juan ${ }^{2 *}$, Zhang Huo-Li ${ }^{3^{*}}$ \\ ${ }^{1}$ College of Chemistry and Chemical Engineering, Liaoning Normal University, Dalian 116029, P.R. \\ China, ${ }^{2}$ Liaoning University of Traditional Chinese Medicine, Shenyang 110032, P.R. China \\ ${ }^{3}$ College of Pharmacy, Dalian Medical University, Dalian 116044, P.R. China \\ *E-mail: dlzhanghl@126.com \\ $\dagger$ Authors contributed equally to this work.
}

\begin{abstract}
The objective of the study was to determine the contents of three active components in Jiejia tincture by establishing HPLC method. Test articles were prepared by ultrasonic extraction. Separation was performed using a Kromasil C18 $(250 \mathrm{~mm} \times 4.6 \mathrm{~mm}, 5 \mu \mathrm{m})$ chromatographic column, and gradient elution was performed with acetonitrile- $0.3 \%$ phosphoric acid solution as the mobile phase at a volumetric flow rate of $0.80 \mathrm{~mL} / \mathrm{min}$. The contents of catechin, baicalin and berberine in Jiejia tincture were determined at the wavelength of $276 \mathrm{~nm}$ and a column temperature of $30 \square$. The results revealed that catechin showed a good linear relationship at the range of $100 \sim 800 \mu \mathrm{g} / \mathrm{mL}$ ( $\mathrm{r}=0.9997)$; baicalin showed a good linear relationship at the range of $15 \sim 120 \mu \mathrm{g} / \mathrm{mL}(\mathrm{r}=0.9996)$, and berberine at the range of $7 \sim 56 \mu \mathrm{g} / \mathrm{mL}(\mathrm{r}=0.9995)$. Their average recovery rates were $99.67 \%$ (RSD $1.01 \%, n=6), 98.7 \%$ (RSD $1.93 \%, n=6)$ and $100.5 \%$ (RSD 2.88\%, $n=6$ ) respectively. The study concluded that the high-performance liquid chromatography established in this study was simple, accurate and reproducible, and can also be used in the determination of catechin, baicalin and berberine contents in Jiejia tincture.
\end{abstract}

Key words: Jiejia tincture, catechin, baicalin, berberine, HPLC

\section{Introduction}

The Jiejia tincture is composed of $100 \mathrm{~g}$ of Acacia catechu, Scutellaria baicalensis and Cortex Phellodendri each, and 30-50 $\mathrm{g}$ of borneol ( $\mathrm{Hu}, 1989)$. It has the heat-clearing and astringing, bleeding-arresting and sore-closing, granulationpromoting and pain-relieving efficacies. And it is commonly used in the clinical treatment of burns and scalds. Acacia catechu is a plant in genus Acacia, family Fabaceae. Its pharmacological effects are astringing dampness, promoting granulation and closing sores, and is mainly used in the clinic for treatment of ulcers, skin eczema, traumatic bleeding, etc. (Chinese Pharmacopoeia Commission, 2010). In recent years, it is also commonly used for anti-oxidation (Osaman, 2011) and anti-bacteria (Li et al, 2001). Scutellaria baicalensis is a plant in the Lamiaceae family, which has the antipyretic, antiinflammatory, anti-allergic, anti-endotoxin, and anti-anxiety efficacies (Liao et al, 2003; Mi et al, 2010). Phellodendron is a Rutaceae family plant. Its pharmacological effects include clearing heat and drying dampness, purging fire and removing toxin, pathogenic microorganism, anti-inflammation and anti-allergy. It is also relatively effective on eczema, burns, bedsores and insect dermatitis (Shen, 2011; Bai, 2008). In recent years, Jiejia tincture is commonly used in the clinic for skin abrasions and severe pressure sores (Gao et al, 2012; He, 2009). However, even with all these efficacies, the method for determination of catechin, baicalin and berberine contents in Jiejia tincture has never been reported. This study established HPLC method for simultaneous determination of catechin, baicalin and berberine contents in Jiejia tincture.

\section{Instruments and reagents} Instruments

The instruments used for the study include the following: high performance liquid chromatograph-UV3000 UV-Vis detector-P3000 high pressure constant flow pump (Beijing Chuangxintongheng Science \& Technology Co., Ltd.); FA1004 analytical balance (Shanghai Balance Instrument Factory); KQ-600DE numerical control ultrasonic cleaner (Kunshan Ultrasonic Instruments Co., Ltd.); and 2100A rotary evaporator (Shanghai Yarong Biochemical Instrument Factory).

\section{Reagents}

The reagents include thus: acetonitrile (HPLC grade, Sigma); phosphoric acid (analytical grade, Tianjin Kemiou Chemical Reagent Co., Ltd.); methanol (analytical grade, Tianjin Kemiou Chemical Reagent Co., Ltd.); catechin standard (batch No. 110877-200001, National Institute for Pharmaceutical and Biological Products Inspection); baicalin standard (batch No. 110715-201016, National Institute for Pharmaceutical and Biological Products Inspection); and berberine standard (batch No. 110713-200911, National Institute for Pharmaceutical and Biological Products Inspection) 


\section{http://dx.doi.org/10.4314/ajtcam.v10i5.25}

\section{Methods and results}

Chromatographic conditions

Kromasil C18 $(250 \mathrm{~mm} \times 4.6 \mathrm{~mm}, 5 \mu \mathrm{m})$ chromatographic column was gradient eluted with acetonitrile- $0.3 \%$ phosphoric acid solution as the mobile phase. The gradient elution mode is shown in Table 1 (collection time was $45 \mathrm{~min}$ ). Volumetric flow rate was $0.80 \mathrm{~mL} / \mathrm{min}$, column temperature was $30 \square$, and detection wavelength was $276 \mathrm{~nm}$. Determination was performed under the above chromatographic conditions. The peak retention time of catechin, baicalin and berberine was consistent with the chromatographic peak retention time of corresponding reference substances. Other components were not interferential on the determination. The chromatogram is shown in Fig.1.

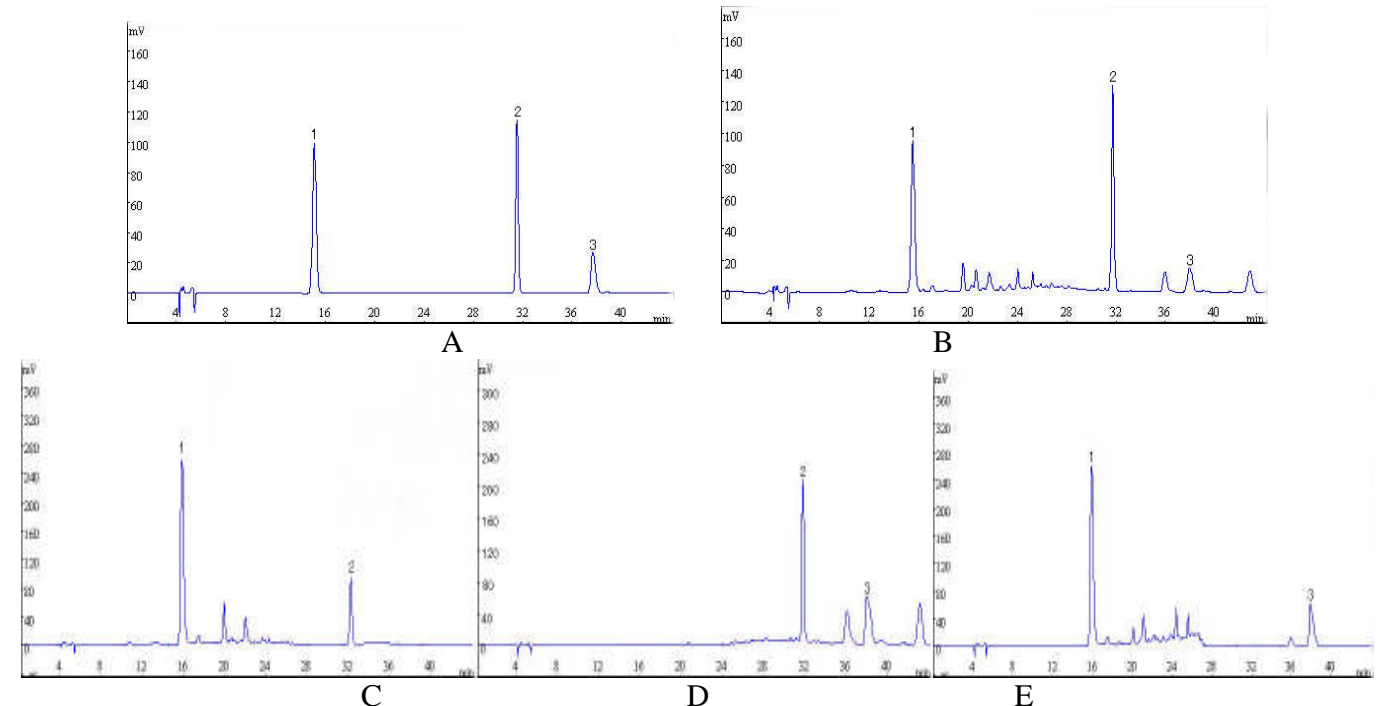

Figure 1: HPLC chromatograms of reference substances (A), samples (B), blank samples without phellodendron amurense $\operatorname{Rupr}(\mathrm{C})$, without Acacia catechu (D), without Scutellaria baicalensis Georgi (E)

Table 1: Gradient elution mode of mobile phase

\begin{tabular}{lll} 
& Table 1: Gradient elution mode of mobile phase \\
\hline Time (min) & Acetonitrile (\%) & $0.3 \%$ phosphoric acid solution $(\%)$ \\
\hline 0 & 10 & 90 \\
5 & 10 & 90 \\
15 & 18 & 82 \\
\hline
\end{tabular}

\section{Preparation of reference solutions}

Adequate amount of Jiejia tincture powder i.e. catechin, baicalin and berberine were precisely weighed respectively, dissolved with $10 \%$ acetonitrile and diluted to volume to prepare into mixed solutions with concentrations of $3 \mathrm{mg} / \mathrm{mL}, 45$ $\mathrm{mg} / \mathrm{mL}$ and $0.21 \mathrm{mg} / \mathrm{mL}$ respectively as the mixed stock solutions of reference substances. A certain volume of mixed reference substances were accurately weighed, stored in volumetric flasks, and added with the mobile phase to dilute into mixed reference solutions with a series of mass concentrations.

\section{Preparation of test solutions}

$5 \mathrm{~g}$ of ground Acacia catechu, Scutellaria baicalensis and Cortex Phellodendri were weighed respectively, added with 10 -fold amount of $80 \%$ ethanol, and ultrasonically extracted at $30 \square$ for 45 min once. The power was $100 \mathrm{kwh}$. After suction filtration, the filtrates were evaporated to dryness in water bath. Then, a certain amount of the powders was weighed and prepared into a concentration of $2 \mathrm{mg} / \mathrm{mL}$ with $10 \%$ acetonitrile. Before injection, the samples were filtered with $0.45 \mu \mathrm{m}$ Millipore membrane, and the test solutions were thereby obtained.

\section{Preparation of negative control solutions}

According to the proportion of prescriptions in the test solutions, medicinal materials in addition to the Acacia catechu, Scutellaria baicalensis and Cortex Phellodendri, were weighed and made into samples. Negative sample solutions were obtained according to the method for preparation of test solution.

\section{Plotting of standard curves}

The pre-prepared mixed reference solutions were precisely weighed, where 1.0, 2.0, 4.0, 5.0, 7.0 and $8.0 \mathrm{~mL}$ were aspirated respectively and diluted to the volume of $10 \mathrm{~mL}$. After filtration through a $0.45 \mu \mathrm{m}$ Millipore membrane, $10 \mu \mathrm{L}$ were taken and injected in accordance with the chromatographic conditions described in 2.1 respectively. Peak areas were 
measured. Standard curves were plotted with peak area A as the ordinate, and mass concentration $\mathrm{C}$ as the abscissa to obtain the regression equation of catechin to be $\mathrm{A}=5140.7 \mathrm{C}+97290$ ( $\mathrm{r}=0.9997)$; regression equation of baicalin to be $\mathrm{A}=24579 \mathrm{C}+38949(\mathrm{r}=0.9995)$; and regression equation of berberine $\mathrm{A}=24579 \mathrm{C}+38949(\mathrm{r}=0.9995)$, indicating that the catechin, baicalin and berberine were in good linear relationship with the peak areas in the mass concentration ranges of $100 \sim 800 \mu \mathrm{g} / \mathrm{mL}, 15 \sim 120 \mu \mathrm{g} / \mathrm{mL}$ and $7 \sim 56 \mu \mathrm{g} / \mathrm{mL}$ respectively.

\section{Precision test}

The mixed reference solutions were taken and continuously and repeatedly injected six times to measure the peak areas respectively. The calculation showed that the RSD of peak areas of catechin, baicalin and berberine was $1.41 \%, 0.78 \%$ and $1.52 \%$ respectively, indicating that the instruments were of good precision.

\section{Repeatability test}

The Jiejia tincture powder of the same batch was accurately weighed in 6 copies, prepared in accordance with the method in 2.3, and injected and measured in accordance with the chromatographic conditions in 2.1. The RSD of peak areas of catechin, baicalin and berberine was calculated to be $1.4 \%, 1.9 \%$ and $2.6 \%$ respectively, indicating that this method has good repeatability.

\section{Stability test}

The same test solution was taken, injected and measured once every $2 \mathrm{~h}$. The injection was repeated a total of 6 times. Peak areas were measured, and RSD of peak areas of catechin, baicalin and berberine was calculated to be $2.4 \%$, $1.7 \%$ and $1.3 \%$ respectively, indicating that the test solution was basically stable within $10 \mathrm{~h}$.

\section{Recovery test}

The Jiejia tincture powders were accurately weighed in 6 copies and prepared in accordance with the method in 2.3. Each copy was precisely added with a certain amount of catechin, baicalin and berberine reference stock solutions, and then measured according to the chromatographic conditions in 2.1 , followed by calculation of contents. The sample recovery rates $(\mathrm{n}=6)$ of catechin, baicalin and berberine were $99.67 \%(\mathrm{R}=1.01 \%), 98.7 \%(\mathrm{R}=1.93 \%)$ and $100.5 \%(\mathrm{R}=2.88 \%)$ respectively. The results of recovery test are shown in Table 2.

Table 2: Results of recovery test

\begin{tabular}{|c|c|c|c|c|c|c|}
\hline \multirow[b]{2}{*}{ Component } & \multirow[b]{2}{*}{$\begin{array}{l}\text { Original } \\
\text { amount/ } \\
\mu \mathrm{g}\end{array}$} & \multirow[b]{2}{*}{$\begin{array}{l}\text { Added } \\
\text { amount/ } \\
\mathrm{Mg}\end{array}$} & \multirow[b]{2}{*}{$\begin{array}{l}\text { Measured } \\
\text { amount/ } \\
\mathrm{Mg}\end{array}$} & \multirow[b]{2}{*}{$\begin{array}{l}\text { Recovery/ } \\
\%\end{array}$} & \multirow[b]{2}{*}{$\begin{array}{l}\text { Average } \\
\text { recovery/ } \\
\%\end{array}$} & \multirow[b]{2}{*}{$\begin{array}{l}\mathrm{RSD} / \\
\%\end{array}$} \\
\hline & & & & & & \\
\hline \multirow{6}{*}{ Catechin } & 203.3 & 200 & 404.39 & 100.55 & \multirow{8}{*}{99.67} & \multirow{8}{*}{1.01} \\
\hline & 202.8 & 200 & 401.02 & 99.11 & & \\
\hline & 201.6 & 200 & 399.56 & 98.98 & & \\
\hline & 200.4 & 200 & 397.13 & 98.37 & & \\
\hline & 203.7 & 200 & 403.88 & 100.09 & & \\
\hline & 200.1 & 200 & 401.96 & 100.93 & & \\
\hline \multirow{7}{*}{ Baicalin } & 77.65 & 75 & 158.92 & 108.36 & & \\
\hline & 75.89 & 75 & 157.35 & 108.61 & & \\
\hline & 76.55 & 75 & 156.76 & 106.95 & \multirow{4}{*}{106.7} & \multirow{5}{*}{1.93} \\
\hline & 75.42 & 75 & 155.49 & 106.76 & & \\
\hline & 77.31 & 75 & 157.52 & 106.95 & & \\
\hline & 75.12 & 75 & 152.28 & 102.88 & & \\
\hline & 15.17 & 14 & 29.18 & 100.07 & \multirow{6}{*}{100.5} & \\
\hline \multirow{5}{*}{ Berberine } & 15.42 & 14 & 30.08 & 104.71 & & \multirow{5}{*}{2.88} \\
\hline & 14.53 & 14 & 28.52 & 99.93 & & \\
\hline & 15.64 & 14 & 29.16 & 96.57 & & \\
\hline & 14.36 & 14 & 28.22 & 0.9900 & & \\
\hline & 14.02 & 14 & 28.43 & 1.0293 & & \\
\hline
\end{tabular}

\section{Sample determination}

The samples were accurately weighed in triplicate respectively, and prepared into solutions according to the method for preparation of test solutions described in 2.3. $10 \mu \mathrm{L}$ were precisely aspirated and determined under the above chromatographic conditions. The peak areas were recorded, and were then substituted into the regression equations to calculate the catechin, baicalin and berberine contents in the samples. The results are shown in Table 3 . 


\section{Discussion}

During the experiment, two mobile phase systems of phosphoric acid-methanol (Zhang \& Zhou, 2009) and phosphoric acid-acetonitrile ( $\mathrm{Li}, 2004)$ were investigated. It was found that in the phosphoric acid-methanol system, the peak of berberine was relatively wide and tailing, and the retention time of baicalin was relatively long. While in the phosphoric acid-acetonitrile system, not only can each test component be effectively separated but the retention time was also shortened, and this had the stable, efficient and economical advantages.

Table 3: Determination results of samples $(n=3)$

\begin{tabular}{ccccccc}
\hline $\begin{array}{c}\text { Sample } \\
\text { ID }\end{array}$ & $\begin{array}{c}\text { Catechin } \\
\text { content/ } \\
(\mathrm{mg} / \mathrm{g})\end{array}$ & RSD/\% & $\begin{array}{c}\text { Baicalin content/ } \\
(\mathrm{mg} / \mathrm{g})\end{array}$ & $\begin{array}{c}\text { RSD/ } \\
\%\end{array}$ & $\begin{array}{c}\text { Berberine } \\
\text { content/ } \\
(\mathrm{mg} / \mathrm{g})\end{array}$ & $\begin{array}{c}\text { RSD/ } \\
\%\end{array}$ \\
\hline 1 & $89.7289 \pm 0.0253$ & 1.35 & $16.3256 \pm 0.0156$ & 1.67 & $4.5733 \pm 0.0112$ & 1.55 \\
2 & $86.8819 \pm 0.0172$ & 1.94 & $16.9610 \pm 0.0092$ & 2.01 & $4.3490 \pm 0.0098$ & 1.32 \\
3 & $87.6386 \pm 0.0204$ & 1.73 & $16.0018 \pm 0.0103$ & 1.82 & $4.145 \pm 0.01085$ & 1.47 \\
\hline
\end{tabular}

In the 2010 edition of Pharmacopoeia, the detection wavelength for catechin and baicalin is recorded to be $280 \mathrm{~nm}$, and the detection wavelength for verberine $265 \mathrm{~nm}$. Relevant literatures have reported that baicalin and berberine can be detected simultaneously at $275 \mathrm{~nm}$ (Jin et al, 2009); catechin and berberine can be detected simultaneously at $270 \mathrm{~nm}$ (Ruan \& Yuan, 2010), and catechin and baicalin at $278 \mathrm{~nm}$ (Tan et al, 2008). Above all, 200 500 nm were selected for wavelength scanning in this experiment, and the results revealed that catechin, baicalin and berberine had maximum absorptions at 276 nm.

The Jiejia tincture is regarded as a good drug for the treatment of burns, scalds and wounds for its widespread medicinal resource, simple preparation, cost-effectiveness, safety and ease of use. It had once been frequently used in hospitals. However, its active components are unknown, and its quality is difficult to control, bringing about problems in clinical medication. This paper established HPLC method for simultaneous determination of the contents of three effective components in the Jiejia tincture. This method has not been reported previously, and is simple in operation, reliable in results, and can be used in the quality control of Jiejia tincture. At the same time, it also laid the foundation for the safe and rational use of the medicine in the clinic.

\section{Acknowledgements}

This work is supported in part by a grant from the Science and Technology of Shenyang (F12-277-1-63), the National Natural Science Foundation of China (No. 81102791), and the Department of Education of Liaoning, China (No. L2012378).

\section{References}

1. Bai Jing. (2008). Observation on 90 cases of eczema treated by Cortex Phellodendri capsule. Journal of Practical Medical Techniques, 15 (27): 3704-3705.

2. Chinese Pharmacopoeia Commission (2010) Pharmacopoeia of the People's Republic of China. China Medical Science Press: 9.

3. Gao Ji-feng, Zhang Ling, Yao Su-yu. (2012). Observation on the clinical efficacy of Jiejia tincture in the treatment of severe pressure sores. Journal of Clinical Pulmonary Medicine, 17(8): 1549-1550.

4. He You-yun. (2009). Application of Zhijia tincture for patients with skin abrasions. Chinese Nursing Research, 23 (3): 697.

5. Hu Xi-ming. (1989). A Collection of Secret Recipes of Chinese Medicines. Wenhui Press,: 150.

6 Jin Liang, Li Ya-nan, Zhang Qiu-yan, Bai ge, Cui Han - ming. (2009). Determination of Baicalin and Berberine in Lianmeiyin preparation. Modern Medicine Journal of China, 11 (12): 32-43.

7. Liao Jyh-Fei, Wen-Yuan Hung, Chien-Fu Chen. (2003). Anxiolytic-like effects of baicalein and baicalin in the Vogel conflict test in mice. European Journal of Pharmacology,464 (2-3):141-146.

8. Li Yi, Xu Xu. (2004). Study on the precipitation reaction between baicalin and berberine by HPLC. Journal of Chromatography B, 810 (1):165-168.

9. Li Zhong-xing, Wang Xiu-hua, Yue Yun-sheng, Zhao Bao-zhen, Chen Jing-bo, Li Ji-hong. (2001).Study on in vitro antibacterial activity of Acacia catechu on 308 clinical strains by a new method. Chinese Journal of Information on Traditional Chinese Medicine, 8(1): 38-39. 
10. Mi-young Yun, Jae-Heon Yang, Dae-Keun Kim, Kwang-Jo Xheong, Hyang-Hee Song, Dong-Hee Kim, Kyu-Jin Cheong, Young-I1 Kim, Song-Chul Shin. (2010). Therapeutic effects of Baicalein on atopic dermatitis-like skin lesions of NC/Nga mice induced by dermatophagoides pteronyssinus. International Immunopharmacology, 10 (9): 1142-1148.

11. Osaman A.M. (2011). Multiple pathways of the reaction of 2,2-diphenyl-1-picrylhydrazyl radical(DPPH) eith (+)-catechin: Evidence for the formation of a cocalent adduct between DPPH and the oxidized form ho the polyphenol.Biochemical and Biophysical Research Communications,412 (3):473-478.

12. Ruan Guiping, Yuan Xujiang. (2010). Simultaneous Determination of Catechin and Berberine Hydrochloride in Zhuhuang Chuihou Powder by HPLC. Traditional Chinese Drug Research and Clinical Pharmacology, 21 (3): 296-298.

13. Shen Ying-jun. (2011). Pharmacology of Chinese Medicine.206-209.

14. Tan S.J., Liu G., Zhang F., Pan MX, Zhang, H, Jiang R. (2008). HPLC determination of catechin,epicatechin and baicalin in Chahuang tincture. Pharmaceutical Analysis, 28 (9):1474-1477.

15. Zhang Ting, Zhou Ping-lan. (2009). Determination of Baicalin and Berberine Hydrochloride in Compound Qinbo Granules by HPLC. Traditional Chinese Drug Research and Clinical Pharmacology, 20 (4): 349-351. 